\title{
SENSIBILIDADE DE PARÂMETROS DA ESTRUTURA NA ANÁLISE DE DESEMPENHO DE PAVIMENTOS FLEXÍVEIS
}

\section{SENSITIVITY OF STRUCTURE PARAMETERS IN FLEXIBLE PAVEMENT PERFORMANCE ANALYSIS}

Karen Amanda Barbosa da Silva; Guilherme Crepaldi Camarini; Heliana Barbosa Fontenele

Universidade Estadual de Londrina - UEL, PR.

E-mail: karen.amanda.b.s@uel.br; guilherme.camarini@uel.br; heliana@uel.br

RESUMO - O dimensionamento de estruturas de pavimentos asfálticos é um processo que envolve o conhecimento de variáveis de difícil previsão e modelagem. A análise de sensibilidade aplicada à previsão de desempenho de pavimentos é essencial para determinar as variáveis mais influentes, e possibilitar a otimização do processo de dimensionamento. $\mathrm{O}$ objetivo deste estudo foi analisar a sensibilidade do desempenho de pavimentos flexíveis às variações nos parâmetros de projeto relativos às espessuras do revestimento, da base e da sub-base e aos módulos de resiliência do revestimento, da base, da sub-base e do subleito. Para tanto, conduziu-se um experimento fatorial fracionário, que teve como variáveis resposta os danos acumulados na estrutura do pavimento, em relação aos defeitos trincas por fadiga e afundamento em trilha de roda. Para obtenção dos danos acumulados, utilizou-se o método empírico-mecanístico de dimensionamento, por meio do programa PerRoad. Concluiu-se que a espessura do revestimento é o parâmetro mais sensível em relação aos defeitos estudados, possuindo maior sensibilidade para defeito trinca por fadiga e uma sensibilidade ligeiramente menor para defeito afundamento em trilha de roda.

Palavras-chave: Método empírico-mecanístico; Análise Fatorial; Defeitos.

ABSTRACT - The design of asphalt pavement structures is a process that involves the knowledge of variables that are difficult to predict and model. Sensitivity analysis applied to pavement performance prediction is essential to determine the most influential variables, and enable the optimization of design process. This study aimed to analyze the sensitivity of flexible pavements performance to variations in design parameters related to the thicknesses of asphalt layer, base and sub-base and resilient modulus of asphalt layer, base, sub-base and subgrade. For this purpose, a fractional factorial experiment was conducted, which had as output variables the accumulated damage in pavement structure, related to fatigue cracking and rutting in wheel wander. To obtain the accumulated damage, mechanistic-empirical design method was used, through PerRoad software. It was concluded that asphalt layer thickness is the most sensitive parameter considering the distresses studied, having a higher sensitivity for fatigue cracking and a slightly lower sensitivity for rutting. Keywords: Mechanistic-empirical method; Factorial analysis; Distress. 


\section{INTRODUÇÃO}

O dimensionamento de estruturas de pavimentos asfálticos é um processo que envolve o conhecimento de variáveis de difícil previsão e modelagem, tais como as características dos materiais de pavimentação, o comportamento deles em relação à aplicação das cargas, o próprio carregamento e o tipo de resposta da estrutura para suportar as cargas sob todas as variações das condições climáticas durante o período de análise (FRANCO, 2007).

Os métodos de dimensionamento empíricos-mecanísticos, com auxílio de programas computacionais, buscam unir todas essas variáveis, com base em estudos teóricos e dados de laboratório ou campo, a fim de se dimensionar uma estrutura mais realista possível, para suportar as condições a qual ela estará sujeita no campo.

A análise de sensibilidade contribui para esse dimensionamento, ao identificar quais os dados de entrada do projeto que mais influenciam no desempenho do pavimento, indicando aos projetistas onde são necessários esforços adicionais para garantir uma maior vida útil ao pavimento (SCHWARTZ et al., 2013).

Este estudo tem por objetivo analisar a sensibilidade das respostas estruturais do pavimento à variação dos parâmetros de projeto relativos às espessuras do revestimento, da base e da sub-base e aos módulos de resiliência do revestimento, da base, da sub-base e do subleito.

\section{DIMENSIONAMENTO DE PAVIMENTOS FLEXÍVEIS}

Nos últimos anos, o dimensionamento de pavimentos flexíveis era realizado com base em métodos empíricos, contudo, esses métodos são calibrados a partir de observações e experiência com certos tipos de pavimentos. O Método do DNER (Departamento Nacional de Estradas de Rodagem), por exemplo, foi desenvolvido com base no método da American Association of State Highway and Transportation Officials (AASHTO). Essa metodologia gera incertezas, pois, esses métodos não podem ser generalizados com confiabilidade para outras condições senão àquelas para as quais eles foram desenvolvidos (FRANCO, 2007).

Com base nisso, atualmente, vêm sendo utilizados métodos de dimensionamento empírico-mecanísticos, que conseguem conciliar os conhecimentos teóricos com os estudos de campo ou de laboratório.
O método de dimensionamento empírico-mecanístico de pavimentos, teve início com o Projeto 1-37A do National Cooperative Highway Research Program (NCHRP), nos Estados Unidos, patrocinado pela AASHTO. O Projeto começou em 1996, sendo disponibilizado um software de teste em 2004, que foi denominado de Mechanistic Empirical Pavement Design Guide (MEPDG), que hoje corresponde ao software AASHTOware M-E Pavement Design ${ }^{\circledR}$ (NCHRP, 2004).

Esse método utiliza um software computacional que permite uma abordagem de análise integrada do tráfego, clima e estrutura do pavimento, para prever os danos acumulados durante a vida útil do pavimento. Nele ainda é possível considerar uma análise especial de cargas e eixos, denominados de espectros de carga por eixo. Com isso, pode-se comparar o comportamento do pavimento com os critérios de desempenho estabelecidos para o projeto.

Segundo Alexandre et al. (2015), as principais deteriorações que ocorrem no pavimento e interferem, significativamente, na vida útil do mesmo, são as trincas por fadiga e o afundamento em trilhas de roda.

$O$ defeito trinca por fadiga consiste em um processo de deterioração estrutural, onde o material perde resistência, quando solicitado, repetidamente, por uma carga, gerando as trincas. A ocorrência desse defeito em pavimentos flexíveis está relacionada com a deformação máxima de tração que ocorre na parte inferior da camada asfáltica, quando da passagem das cargas do tráfego (FRANCO, 2007).

$O$ defeito afundamento em trilhas de roda na superfície do pavimento é consequência das deformações plásticas que ocorrem ao longo das diferentes camadas que constituem a estrutura. Esse defeito pode levar ao comprometimento estrutural do pavimento e, também elevar o potencial para a ocorrência do fenômeno da hidroplanagem (FRANCO, 2007).

Ainda segundo Franco (2007), a avaliação do afundamento em trilhas de roda ocorre de maneira indireta, com base nas tensões verticais de compressão no topo do subleito, sendo que as deformações nas demais camadas são consideradas insignificantes.

Existem diferentes programas computacionais que podem ser utilizados para dimensionar os pavimentos, como por exemplo: o AASHTOWare, Medina, PerRoad, Weslea, entre outros. 
Neste estudo, para o dimensionamento do pavimento, utilizou-se o programa Perpetual Pavement Design Software (PerRoad). Este software utiliza a teoria linear elástica de múltiplas camadas para dimensionar $\mathrm{o}$ pavimento. O PerRoad ainda possibilita uma abordagem probabilística, permitindo a variação de parâmetros de estrutura e de tráfego na previsão do desempenho do pavimento.

No PerRoad, primeiramente é necessário fornecer os dados de entrada, sendo eles: material e espessura das camadas; módulo de resiliência e coeficiente de Poisson das camadas e do subleito; condições climáticas e características do tráfego. O programa ainda tem a opção de calcular o módulo de resiliência da camada asfáltica de acordo com a temperatura do ar, que muda de acordo com a estação do ano. Posteriormente, faz-se a análise estatística do pavimento para obter as tensões e deformações.

\section{ANÁLISE DE SENSIBILIDADE}

A análise de sensibilidade consiste na verificação de quais parâmetros de entrada de um modelo (numérico ou não), influenciam significativamente os resultados de saída desse modelo ( SALTELLI et al., 2008). Na análise de pavimentos, a sensibilidade é útil para identificar os parâmetros que mais influenciam no desempenho de pavimentos e para verificar a adequabilidade dos algoritmos de dimensionamento (SCHWARTZ et al., 2013).

A análise de sensibilidade pode ser feita de dois modos: perante uma análise local ou mediante uma análise global. A primeira consiste na variação de um parâmetro por vez, sendo o processo repetido até que todos os parâmetros de interesse tenham sido verificados. Já a análise global permite verificar a interação entre as diversas variáveis independentes no resultado final, pois, todos os parâmetros de entrada são variados simultaneamente.

Um dos benefícios do uso de uma análise global consiste na possibilidade de analisar todas as combinações possíveis entre os fatores, contudo, dependendo da quantidade de parâmetros a serem variados, tem-se um enorme número de simulações, o que inviabiliza esse tipo de análise (SALTELLI et al., 2008).

No entanto, é possível selecionar uma fração dessas combinações para gerar uma análise menor, fácil de ser aplicada e que produz resultados úteis, sendo este tipo de planejamento denominado de análise fatorial fracionária.

As análises fracionárias são divididas em resoluções. Segundo Montgomery (2012), os principais tipos são as resoluções III, IV e V, sendo que cada uma possui uma quantidade de fatores que geram um determinado número de combinações.

Conhecer a sensibilidade de uma variável no dimensionamento do pavimento permite determinar em qual parâmetro intervir, caso a estrutura dimensionada não atenda aos critérios de serviço. Isso acarreta em um menor tempo de dimensionamento da estrutura, além de uma melhor utilização dos recursos disponíveis, tanto naturais, com a diminuição do consumo de materiais granulares, quanto financeiros, com a diminuição da manutenção e reabilitação de pavimentos e com baixos custos de operação dos veículos.

Atualmente, diversos fatores contribuem para a perda de desempenho de um pavimento, entre eles: fatores ambientais, climáticos, a qualidade dos materiais, a qualidade da execução e as cargas devidas ao tráfego. De um modo geral, as principais deteriorações no pavimento são as trincas por fadiga e o afundamento em trilhas de roda (ALEXANDRE et al., 2015).

Diversos estudos foram realizados a respeito da análise de sensibilidade de parâmetros de pavimentos flexíveis, entre eles, têm-se os estudos de Vidotto e Fontenele (2013) e Pelisson et al. (2015), que realizaram uma análise de sensibilidade local, e concluíram que a espessura do revestimento influencia significativamente as respostas estruturais, sendo os fatores de danos referentes ao defeito trinca por fadiga mais sensíveis que os fatores referentes ao defeito afundamento nas trilhas de roda.

Tal fato confirma as evidências de estudos anteriores, da importância da espessura da camada asfáltica, inclusive os que levaram à adoção, por parte da AASHTO (1986), de espessura mínima de $12,5 \mathrm{~cm}$ para o revestimento asfáltico de rodovias com tráfego médio. Contudo, no Brasil, isso tem sido ignorado, com adoção de espessuras que, se representam alguma economia no custo de construção, resultam em custos altíssimos de manutenção e reabilitação e, mais ainda, de operação de veículos ao longo da vida em serviço (VIDOTTO; FONTENELE, 2013; PELISSON et al., 2015). 
Li et al. (2012), desenvolveram uma análise de sensibilidade local das propriedades dos materiais granulares, referentes à base e ao subleito, eles concluíram que o módulo de resiliência da base e do subleito e a espessura da base são as propriedades que apresentaram maior influência na previsão de desempenho em todos os critérios, independente do volume de tráfego, e o coeficiente de Poisson da base e do subleito também apresentaram sensibilidade.

Outro estudo desenvolvido nessa área foi o de Schwartz et al. (2013), que realizaram uma análise de sensibilidade global, aplicando uma técnica de amostragem randômica (Latin Hypercube Sampling). Foram 23 parâmetros investigados, sob 15 cenários diferentes (combinações de 3 volumes de tráfego com 5 regiões climáticas). 0 estudo resultou em mais de 40 mil simulações no software MEPDG. Ao final, demostraram que os defeitos trincas por fadiga e afundamento nas trilhas de roda são mais sensíveis às propriedades da camada de revestimento asfáltico (espessura, módulo dinâmico, coeficiente de Poisson, volume de vazios e teor de ligante), ao módulo de elasticidade do subleito e módulo de elasticidade da camada de base.

Saad (2014) realizou uma análise de sensibilidade fatorial completa, para verificar a influência do lençol freático e da rigidez da base e do subleito no surgimento do defeito afundamento em trilha de roda, na superfície do pavimento. $\mathrm{O}$ autor concluiu que a presença do lençol freático se torna mais prejudicial ao pavimento quando a rigidez do subleito diminui e que esse efeito não é observado, significativamente, quando se diminui a rigidez da base.

\section{MÉTODOS}

Os procedimentos desta pesquisa foram divididos em duas etapas: a primeira consistiu na determinação dos danos acumulados relativos aos defeitos trinca por fadiga e afundamento nas trilhas de roda, utilizando o software PerRoad v. 4.4. A segunda tratou-se da análise de sensibilidade, aplicando um design fatorial fracionário.

Primeiramente, houve a determinação dos parâmetros de projeto a serem variados, sendo eles: espessura do revestimento (Esp.R), espessura da base (Esp.B), espessura da sub-base (Esp.Sbb), módulo de resiliência do revestimento (Mr.R), módulo de resiliência da base (Mr.B), módulo de resiliência da sub-base (Mr.Sbb) e módulo de resiliência do subleito (Mr.Sbl).

Na Tabela 1, são apresentados os valores dos fatores considerados nesta pesquisa. Tratando-se de um experimento fatorial fracionário de 2 níveis, foram definidos um nível superior e um inferior para cada fator. Os valores na Tabela 1 estão apresentados conforme as unidades de medida do software PerRoad: módulos de resiliência em psi e espessuras em polegadas.

Posteriormente, elaborou-se um design fatorial fracionário de resolução IV, pois, segundo Kleijmen (2005), esse tipo de resolução é o mais adequado para se quantificar os efeitos de primeira ordem.

Tabela 1. Parâmetros variáveis

\begin{tabular}{lrr}
\hline Parâmetros & $\begin{array}{r}\text { Nível Inferior } \\
(-1)\end{array}$ & \multicolumn{1}{c}{$\begin{array}{r}\text { Nível Superior } \\
(+1)\end{array}$} \\
\hline Esp.R & 2,95 pol. & 5,91 pol. \\
Esp.B & 5,91 pol. & 9,06 pol. \\
Esp.Sbb & 7,87 pol. & 11,81 pol. \\
Mr.R & 435.113 psi & 1.015 .264 psi \\
Mr.B & 29.008 psi & 58.015 psi \\
Mr.Sbb & 14.504 psi & 29.008 psi \\
Mr.Sbl & $7.252 \mathrm{psi}$ & 14.504 psi \\
\hline
\end{tabular}

Fonte: Os autores.

Neste delineamento, o número de observações (Obs.) do subconjunto pode ser obtido com base na Equação 1:

$$
\text { Obs. }=N^{k-p}
$$

sendo: $\mathrm{N}$ o número de níveis do fatorial, $\mathrm{k}$ o número de fatores e $\mathrm{p}$ refere-se à fração de análise dos fatores.

Nessa análise fracionária tem-se o número de fatores igual a sete e o nível igual a dois (um valor mínimo e um máximo) conforme observado na Tabela 1; e a fração a ser analisada igual a 2, gerando 32 observações. Os valores de cada parâmetro, em cada observação, são expostos na Tabela 2.

Após a montagem do experimento fatorial, determinou-se os demais dados de entrada a serem fornecidos ao programa PerRoad: clima, características do tráfego e os coeficientes de Poisson das camadas do pavimento. Esses valores foram mantidos fixos durante a análise de sensibilidade. As 
informações referentes ao clima não foram utilizadas nesse estudo, sendo desabilitada no programa a opção de realizar cálculos com base na temperatura média do ar.

Tabela 2. Valores dos parâmetros em cada observação fatorial

\begin{tabular}{|c|c|c|c|c|c|c|c|}
\hline Obs. & $\begin{array}{l}\text { Esp.R } \\
\text { (pol.) }\end{array}$ & $\begin{array}{l}\text { Esp.B } \\
\text { (pol.) }\end{array}$ & $\begin{array}{c}\text { Esp.Sbb } \\
\text { (pol.) }\end{array}$ & Mr.R (psi) & Mr.B (psi) & Mr.Sbb (psi) & Mr.Sbl (psi) \\
\hline 1 & 2,95 & 5,91 & 7,87 & 435.113 & 29.008 & 29.008 & 14.504 \\
\hline 2 & 5,91 & 5,91 & 7,87 & 435.113 & 29.008 & 14.504 & 7.252 \\
\hline 3 & 2,95 & 9,06 & 7,87 & 435.113 & 29.008 & 14.504 & 7.252 \\
\hline 4 & 5,91 & 9,06 & 7,87 & 435.113 & 29.008 & 29.008 & 14.504 \\
\hline 5 & 2,95 & 5,91 & 11,81 & 435.113 & 29.008 & 14.504 & 14.504 \\
\hline 6 & 5,91 & 5,91 & 11,81 & 435.113 & 29.008 & 29.008 & 7.252 \\
\hline 7 & 2,95 & 9,06 & 11,81 & 435.113 & 29.008 & 29.008 & 7.252 \\
\hline 8 & 5,91 & 9,06 & 11,81 & 435.113 & 29.008 & 14.504 & 14.504 \\
\hline 9 & 2,95 & 5,91 & 7,87 & 1.015 .264 & 29.008 & 14.504 & 7.252 \\
\hline 10 & 5,91 & 5,91 & 7,87 & 1.015 .264 & 29.008 & 29.008 & 14.504 \\
\hline 11 & 2,95 & 9,06 & 7,87 & 1.015 .264 & 29.008 & 29.008 & 14.504 \\
\hline 12 & 5,91 & 9,06 & 7,87 & 1.015 .264 & 29.008 & 14.504 & 7.252 \\
\hline 13 & 2,95 & 5,91 & 11,81 & 1.015 .264 & 29.008 & 29.008 & 7.252 \\
\hline 14 & 5,91 & 5,91 & 11,81 & 1.015 .264 & 29.008 & 14.504 & 14.504 \\
\hline 15 & 2,95 & 9,06 & 11,81 & 1.015 .264 & 29.008 & 14.504 & 14.504 \\
\hline 16 & 5,91 & 9,06 & 11,81 & 1.015 .264 & 29.008 & 29.008 & 7.252 \\
\hline 17 & 2,95 & 5,91 & 7,87 & 435.113 & 58.015 & 29.008 & 7.252 \\
\hline 18 & 5,91 & 5,91 & 7,87 & 435.113 & 58.015 & 14.504 & 14.504 \\
\hline 19 & 2,95 & 9,06 & 7,87 & 435.113 & 58.015 & 14.504 & 14.504 \\
\hline 20 & 5,91 & 9,06 & 7,87 & 435.113 & 58.015 & 29.008 & 7.252 \\
\hline 21 & 2,95 & 5,91 & 11,81 & 435.113 & 58.015 & 14.504 & 7.252 \\
\hline 22 & 5,91 & 5,91 & 11,81 & 435.113 & 58.015 & 29.008 & 14.504 \\
\hline 23 & 2,95 & 9,06 & 11,81 & 435.113 & 58.015 & 29.008 & 14.504 \\
\hline 24 & 5,91 & 9,06 & 11,81 & 435.113 & 58.015 & 14.504 & 7.252 \\
\hline 25 & 2,95 & 5,91 & 7,87 & 1.015 .264 & 58.015 & 14.504 & 14.504 \\
\hline 26 & 5,91 & 5,91 & 7,87 & 1.015 .264 & 58.015 & 29.008 & 7.252 \\
\hline 27 & 2,95 & 9,06 & 7,87 & 1.015 .264 & 58.015 & 29.008 & 7.252 \\
\hline 28 & 5,91 & 9,06 & 7,87 & 1.015 .264 & 58.015 & 14.504 & 14.504 \\
\hline 29 & 2,95 & 5,91 & 11,81 & 1.015 .264 & 58.015 & 29.008 & 14.504 \\
\hline 30 & 5,91 & 5,91 & 11,81 & 1.015 .264 & 58.015 & 14.504 & 7.252 \\
\hline 31 & 2,95 & 9,06 & 11,81 & 1.015 .264 & 58.015 & 14.504 & 7.252 \\
\hline 32 & 5,91 & 9,06 & 11,81 & 1.015 .264 & 58.015 & 29.008 & 14.504 \\
\hline
\end{tabular}

Fonte: Próprios autores

Para obtenção das cargas de tráfego atuantes, foram utilizados os dados de espectros de cargas por eixo, desenvolvidos por Fontenele e Fernandes Junior (2014), com base na balança de pesagem da Rodovia dos Imigrantes, no estado de São Paulo. A pressão de enchimento dos pneus é fixa no PerRoad e equivalente a 690 $\mathrm{kPa}$ (100 psi).

Os valores atribuídos aos coeficientes de Poisson de cada camada, também tiveram como base o trabalho de Fontenele e Fernandes Junior
(2014). Na Tabela 3 tem-se os coeficientes utilizados.

Tabela 3. Coeficientes de Poisson utilizados

\begin{tabular}{lc}
\hline \multicolumn{1}{c}{ Parâmetros Fixos } & Valor \\
\hline Coef. de Poisson - revestimento & 0,30 \\
Coef. de Poisson - base & 0,40 \\
Coef. de Poisson - sub-base & 0,40 \\
Coef. de Poisson - subleito & 0,45 \\
\hline Fonte: Fontenele e Fernandes Junior (2014) &
\end{tabular}


O desempenho do pavimento, segundo os defeitos trincas por fadiga e afundamento nas trilhas de roda, foi medido em função do consumo percentual de danos acumulados pela passagem de um milhão de eixos. Para tanto, o PerRoad utiliza de ciclos de Monte Carlo para selecionar randomicamente uma carga de eixo, respeitando os espectros de cargas informados pelo usuário, e calcula a deformação máxima de tração na fibra inferior do revestimento $\left(\varepsilon_{t, n}\right) \mathrm{e}$ deformação máxima de compressão no topo do subleito $\left(\varepsilon_{v, n}\right)$, no enésimo ciclo (TIMM \& NEWCOMB, 2006). Estas respostas foram relacionadas empiricamente com o número de aplicações de cargas de eixo até a falha por fadiga $\left(N_{f, n}\right)$ e afundamento nas trilhas de roda $\left(N_{r, n}\right)$, no enésimo ciclo de Monte Carlo, por meio das Equações 2 e 3, desenvolvidas por Timm e Newcomb (2003).

$$
\begin{aligned}
& N_{f, n}=2,83 * 10^{-6}\left(\frac{1}{\varepsilon_{t, n}}\right)^{3,148} \\
& N_{r, n}=6,026 * 10^{-8}\left(\frac{1}{\varepsilon_{v, n}}\right)^{3,87}
\end{aligned}
$$

Sequencialmente, foram calculados os danos causados a cada ciclo de aplicação de carga de eixo $\left(D_{n}\right)$, pela Equação 4.

$$
D_{n}=\frac{1}{N_{f n}}
$$

Os danos individuais calculados pela Equação 4 foram acumulados com a aplicação da Miner's Hypothesis (PAPAGIANNAKIS \& MASAD, 2008; MONISMITH, 2012 e AASHTO, 2015), por meio da Equação 5 , em que " $m$ " representa a quantidade de ciclos de Monte Carlo. Nesta pesquisa, foram considerados 5.000 ciclos de Monte Carlo.

$$
D=\sum_{n=1}^{m} D_{n}
$$

Depois de acumulado o dano total dos 5.000 ciclos, calculou-se o dano médio $\left(D_{\text {med }}\right)$, pela Equação 6. E, por fim, calculou-se o dano acumulado a cada $10^{6}$ solicitações de cargas de eixos $\left(D_{10^{6}}\right)$, pela Equação 7 , respeitando as frequências relativas (espectros de cargas por eixo) fornecidas no início do processo.

$$
\begin{gathered}
D_{\text {med }}=\frac{D}{m} \\
D_{10^{6}}=D_{\text {med }} \times 10^{6}
\end{gathered}
$$

Os danos acumulados a cada um milhão de eixos, por fadiga e por afundamento, foram considerados como variáveis resposta no experimento fatorial.

Em seguida, para a segunda etapa dessa metodologia, foi utilizado o programa RStudio, onde foram feitas as codificações das 32 observações fatoriais. Posteriormente, inseriu-se no programa essas combinações na forma matricial, para que, juntamente com os resultados obtidos no programa PerRoad, pudesse ser feito o cálculo dos coeficientes fatoriais de cada parâmetro.

\section{RESULTADOS E DISCUSSÕES}

Por meio do programa PerRoad, obtiveram-se os danos acumulados por fadiga e afundamento a cada $10^{6}$ solicitações de cargas de eixos $\left(D_{10^{6}}\right)$, em cada uma das observações geradas pelo experimento fatorial. Estes resultados obtidos estão expostos na Tabela 4.

Posteriormente, com base nos resultados referentes à trinca por fadiga e na matriz codificada das observações, obtiveram-se os coeficientes fatoriais dos parâmetros referentes ao dano por fadiga por um milhão de eixos. $\mathrm{Na}$ Figura 1, tem-se o gráfico dos coeficientes obtidos.

Com base na Figura 1, observa-se que os parâmetros apresentam a seguinte ordem decrescente de sensibilidade: espessura do revestimento, módulo de resiliência do revestimento, módulo de resiliência da base, módulo de resiliência da sub-base, espessura da base, espessura da sub-base e módulo de resiliência do subleito. 
Tabela 4. Danos acumulados em $10^{6}$ solicitações de cargas de eixos

\begin{tabular}{|c|c|c|}
\hline Obs. & $\begin{array}{c}\text { Consumo } \\
\text { Fadiga }\end{array}$ & $\begin{array}{c}\text { Consumo } \\
\text { Afundamento }\end{array}$ \\
\hline 1 & 5,1410 & 1,3011 \\
\hline 2 & 0,9798 & 1,0958 \\
\hline 3 & 5,5767 & 3,2398 \\
\hline 4 & 0,6832 & 0,0869 \\
\hline 5 & 6,2718 & 0,5434 \\
\hline 6 & 0,7207 & 0,3784 \\
\hline 7 & 4,8357 & 0,8271 \\
\hline 8 & 0,7748 & 0,0461 \\
\hline 9 & 1,8531 & 4,7672 \\
\hline 10 & 0,1290 & 0,0568 \\
\hline 11 & 1,2891 & 0,3532 \\
\hline 12 & 0,1571 & 0,2337 \\
\hline 13 & 1,3280 & 1,2286 \\
\hline 14 & 0,1500 & 0,0166 \\
\hline 15 & 1,4632 & 0,1574 \\
\hline 16 & 0,1294 & 0,0925 \\
\hline 17 & 1,5659 & 3,1962 \\
\hline 18 & 0,4142 & 0,1306 \\
\hline 19 & 1,4770 & 0,2877 \\
\hline 20 & 0,3055 & 0,2852 \\
\hline 21 & 1,8807 & 1,8282 \\
\hline 22 & 0,3250 & 0,0527 \\
\hline 23 & 1,3639 & 0,1084 \\
\hline 24 & 0,3398 & 0,1772 \\
\hline 25 & 0,7560 & 0,5267 \\
\hline 26 & 0,0947 & 0,2578 \\
\hline 27 & 0,5242 & 0,8330 \\
\hline 28 & 0,0785 & 0,0073 \\
\hline 29 & 0,5815 & 0,1859 \\
\hline 30 & 0,1092 & 0,1552 \\
\hline 31 & 0,5723 & 0,4814 \\
\hline 32 & 0,0673 & 0,0016 \\
\hline
\end{tabular}

Fonte: Próprios autores

Nota-se que a espessura do revestimento é a mais sensível, apresentando um coeficiente de 0,96 (em módulo), muito elevado em comparação aos demais. Também é possível observar que o módulo de resiliência do revestimento e da base apresentam um coeficiente de sensibilidade significativo, já os demais fatores, apresentaram valores menores que 0,30 (em módulo), não sendo considerados representativos para este defeito. 
Figura 1. Sensibilidade para trinca por fadiga

Fonte: Próprios autores

Figura 2. Sensibilidade para afundamento em trilha de roda

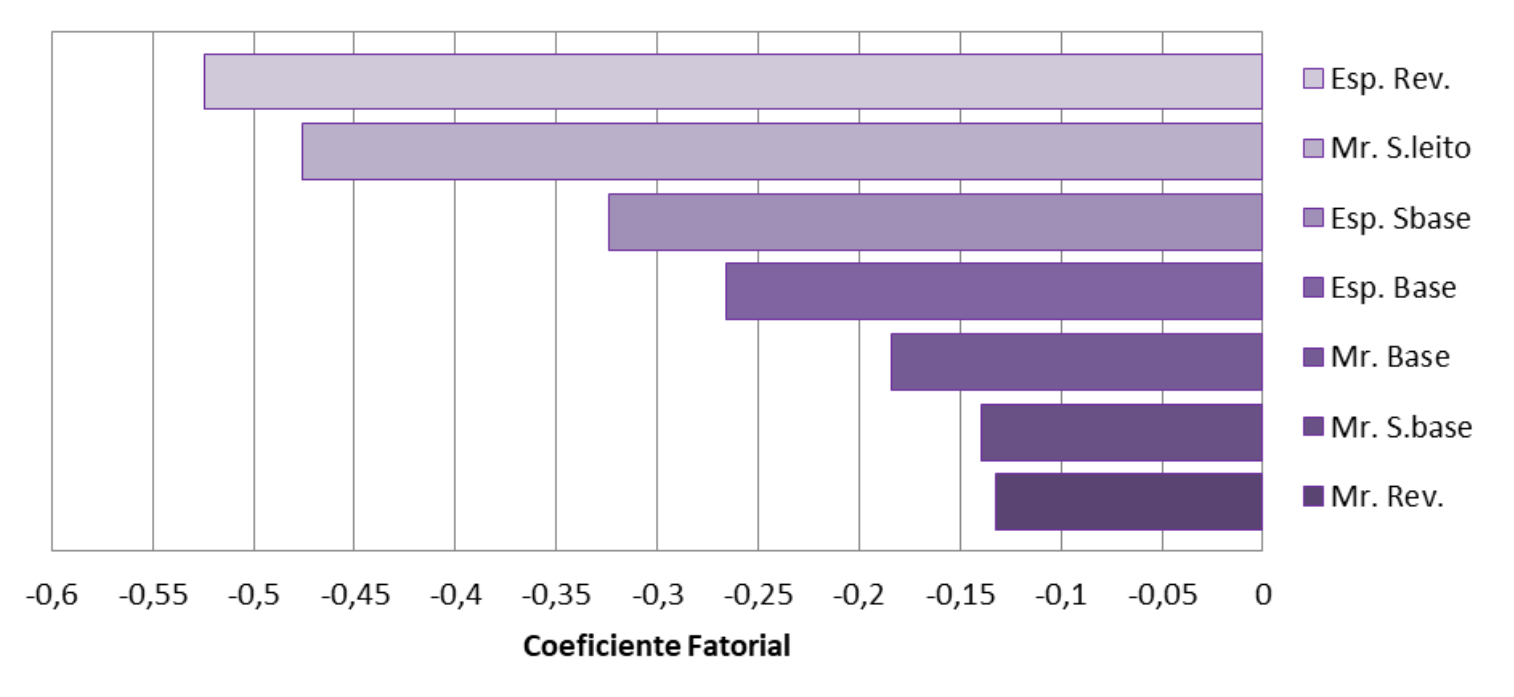

Fonte: Próprios autores

De maneira análoga à sensibilidade por fadiga, com base nos resultados referentes ao afundamento em trilhas de roda, obtiveram-se os coeficientes fatoriais dos parâmetros para o dano acumulado por afundamento a cada um milhão de eixos, sendo os resultados expostos na Figura 2.

Nota-se, na Figura 2, que os parâmetros possuem a seguinte ordem decrescente de sensibilidade: espessura do revestimento, módulo de resiliência do subleito, espessura subbase, espessura base, módulo de resiliência base, módulo de resiliência da sub-base e módulo de resiliência do revestimento.

Assim como para a trinca por fadiga, a espessura do revestimento foi o parâmetro mais sensível, possuindo coeficiente fatorial de 0,52 (em módulo), não sendo tão elevado quanto o valor para trinca por fadiga, mas que demonstra a importância desse parâmetro no dimensionamento da estrutura do pavimento.

Os fatores módulo de resiliência do subleito e espessura da sub-base possuem coeficiente fatorial maior que 0,30 (em módulo), logo, apresentam sensibilidade em relação ao defeito afundamento em trilhas de roda.

Observa-se que todos os coeficientes, da trinca por fadiga e do afundamento da trilha de roda são negativos, isso significa, que ao se utilizar o nível superior do parâmetro (+1), o dano acumulado será menor, logo, o pavimento durará por mais tempo. Contudo, ao se utilizar o nível 
inferior (-1), o dano acumulado será maior, portanto, o pavimento durará menos.

\section{CONSIDERAÇÕES FINAIS}

Com base no exposto, pode-se concluir que a espessura do revestimento é o parâmetro mais sensível em relação aos defeitos estudados, possuindo maior sensibilidade para defeito trinca por fadiga, possuindo uma sensibilidade um pouco menor para o afundamento em trilha de roda.

Isso corrobora com os estudos desenvolvidos na área, sobre a importância desse parâmetro no dimensionamento de pavimentos flexíveis, o que não é levado em consideração no Brasil, pois, segundo o método de dimensionamento do DNIT (2006) a espessura máxima que o revestimento pode ter é de 12,50 centímetros, sendo esta a espessura mínima exigida no método da AASHTO (1986), o que gera altos custos de manutenção e reabilitação dos pavimentos, além dos gastos de operação com os veículos.

Neste estudo, também pode-se verificar que o módulo de resiliência do revestimento e da base também apresentam sensibilidade significativa, em relação ao defeito trinca por fadiga. Já os fatores módulo de resiliência do subleito e espessura da sub-base apresentam sensibilidade em relação ao defeito afundamento em trilhas de roda.

Com base no conhecimento da sensibilidade desses parâmetros, pode-se aprimorar o dimensionamento de pavimentos flexíveis, pois, quando uma determinada estrutura não atingir os critérios de serviço, sabese em qual parâmetro intervir para melhorar o seu desempenho e assim reduzir o tempo de dimensionamento.

Também é possível prolongar a vida útil do pavimento, ao estabelecer as espessuras e propriedades adequadas para cada camada, além de economizar recursos naturais, diminuindo consumo de materiais granulares, e recursos financeiros, com a diminuição da manutenção e reabilitação e operação de veículos.

Em relação à análise de sensibilidade, percebe-se que a variação dos parâmetros pode acarretar mudanças significativas no desempenho do pavimento. Contudo, ainda são necessários estudos mais aprofundados, que levem em consideração outros parâmetros, como: clima, coeficiente de Poisson, etc., bem como uma análise de sensibilidade fatorial completa, com intuito de entender melhor a interação entre os parâmetros, e com isso auxiliar no dimensionamento dos pavimentos.

\section{REFERÊNCIAS}

ALEXANDRE, L. H. C.; ALVES, S. A. M.; BORBA, P. C. S.; RÉUS, T. F.; TEIXEIRA, R.; SILVA JÚNIOR, C. A. P.; FONTENELE, H. B. Sensibilidade dos Pavimentos Flexíveis às Variações dos Módulos de Elasticidade. Revista de Engenharia e Tecnologia, v. 7, n. 1, p. 71-78, 2015.

AMERICAN ASSOCIATION OF STATE HIGHWAY AND TRANSPORTATION OFFICIALS - AASHTO. Guide for Design of Pavement Structures. Washington, D. C., 1986.

AMERICAN ASSOCIATION OF STATE HIGHWAY AND TRANSPORTATION OFFICIALS EXECUTIVE COMMITTEE - AASHTO. Mechanistic-empirical pavement design guide: A manual of practice. 2 . ed. Washington, D. C., 2015.

DEPARTAMENTO NACIONAL DE INFRAESTRUTURA DE TRANSPORTE - DNIT. Manual de Pavimentação. Pub. IPR-719. Rio de Janeiro, 2006.

FONTENELE, H. B.; FERNANDES JÚNIOR, J. L. O Efeito da Caracterização do Tráfego no Desempenho do Pavimento Flexível. Ciência \& Engenharia, v. 23, n. 1, p. p. 09-16, 2014. https://doi.org/10.14393/19834071.2014.24552

FRANCO, F. A. C. P.. Método de dimensionamento mecanístico-empírico de pavimentos asfálticos - SisPav. 2007. Tese (Doutorado) - Universidade Federal do Rio de Janeiro, Rio de Janeiro, 2007.

KLEIJNEN, J. P. C.; SANCHEZ, S. M.; LUCAS, T. W.; CIOPPA, T. M. A user's guide to the brave new world of designing simulation experiments. Informs Journal on Computing, v. 17, n. 3, p.p. 263-289, 2005. https://doi.org/10.1287/ijoc.1050.0136.

LI, R.; SCHWARTZ, C. W.; KIM, S.; CEYLAN, H. Local Sensitivity of Mechanistic-Empirical Flexible Pavement Performance Predictions to Unbound Material Property Inputs. Proceedings, Geo Congress 2012: State of the Art and Practice in Geotechnical Engineering, 2012. https://doi.org/10.1061/9780784412121.154 
MONISMITH, C. L. Flexible Pavement Analysis and Design - A Half Century of Achievement.

In: GEOCONGRESS, 2012. Oakland, California: American Society of Civil Engineers; 2012.

p.

187-220.

https://doi.org/10.1061/9780784412138.0008

MONTGOMERY, D. C. Design and Analysis of Experiments. 8.ed. John Wiley \& Sons Inc., 2012.

NATIONAL COOPERATIVE HIGHWAY RESEARCH PROGRAM - NCHRP. Guide for MechanisticEmpirical Design of New and Rehabilitated Pavement Structures. Final Report NCHRP 1-37 A. Champaign, Illinois, 2004.

PAPAGIANNAKIS, A. T.; MASAD, E. A. Pavement Design and Materials. Hoboken: John Wiley \& Sons, 2008.

PELISSON, N. D.; FERNANDES JÚNIOR, J. L.; SILVA JÚNIOR, C. A. P.; FONTENELE, H. B. Avaliação de Desempenho pelo MEPDG: o Efeito da Variação de Espessuras de Pavimentos Flexíveis. Journal of Transport Literature, v. 9, n. 1, p. 20-24, 2015. https://doi.org/10.1590/2238-1031.jtl.v9n1a4

SAAD, B. Factorial Numerical Analysis of Flexible Pavement Foundations with Emphasis on Groundwater Table Effect. In: CONFERENCE INFORMATION GEO-CONGRESS, 2014 Atlanta, GE: American Society of Civil Engineers, 2014.p. 2931-2941, 2014. https://doi.org/10.1061/9780784413272.284

SALTELLI, A.; RATTO, M.; ANDRES, T.; CAMPOLONGO, F.; CARIBONI, J. GATELLI, D.; SAISANA, M.; TARANTOLA, S. Global Sensitivity Analysis - The Primer. [Nova Jersey]: John Wiley \& Sons, 2008.

https://doi.org/10.1002/9780470725184

SCHWARTZ, W. C., LI, R., CEYLAN, H.; KIM, S.; GOPALAKRISHNAN, K. Global Sensitivity Analysis of Mechanistic-Empirical Performance Predictions for Flexible Pavements. Transportation Research Record: Journal of the Transportation Research Board, n. 2368, Transportation Research Board of the National Academies, Washington, D.C., 2013, pp. 12-23. https://doi.org/10.3141/2368-02
TIMM, D. H.; NEWCOMB, D. E. Calibration of Flexible Pavement Performance Equations for Minnesota Road Research Project. Transportation Research Record: Journal of the Transportation Research Board, n. 1853, p. 134142, 2003. https://doi.org/10.3141/1853-15

TIMM, D. H.; NEWCOMB, D. E. Perpetual pavement design for flexible pavements in the US. International Journal of Pavement Engineering, v. 7, n. 2, p. 111-119, 2006. https://doi.org/10.1080/10298430600619182

VIDOTTO, J. P.; FONTENELE, H. B. Efeito da Variação da Espessura do Revestimento nas Respostas Estruturais do Pavimento Flexível. Semina: Ciências Exatas e Tecnológicas, v. 34, n. 2, p. 155-166, 2013. https://doi.org/10.5433/1679$\underline{0375.2013 v 34 n 2 \mathrm{p} 155}$ 